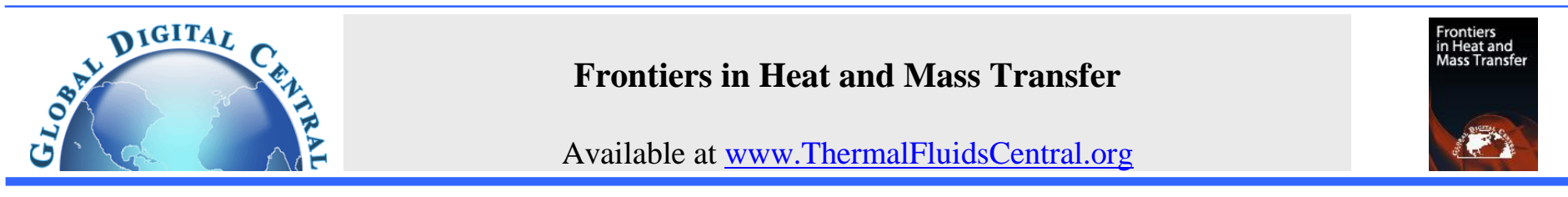

\title{
EXAMINATION OF THERMAL PROPERTIES OF CARBON-CARBON AND GRAPHATIZED CARBON-CARBON COMPOSITES
}

\author{
Melanie Patrick ${ }^{\mathrm{a}}$, Messiha Saad, \\ ${ }^{a}$ North Carolina A\&T State University, Greensboro, NC, 27411, USA
}

\begin{abstract}
Thermal characterization is essential to the proper assignment of composites to specific applications. Specific heat, thermal diffusivity, and thermal conductivity are critical in the engineering design process and in the analysis of aerospace vehicles, space systems, power generation, transportation systems, and energy storage devices including fuel cells. This paper examines the thermal properties through the thickness of Carbon-Carbon and the impact of Graphitization is explored. Following ASTM standards, the Flash Method and Differential Scanning Calorimetry measured thermal diffusivity and specific heat respectively. These measurements and density data allowed for the computation of thermal conductivity.
\end{abstract}

Keywords: Flash Method; Diffusivity; Conductivity; Specific Heat

\section{INTRODUCTION}

Thermal and mechanical characterization of composite materials is the key for appropriate utilization. More often only mechanical properties of a composite are used to deem it suitable for an application, but adding a thermal aspect to this determination yields a stronger verification to the composite's applicability. Thermal diffusivity, specific heat, and thermal conductivity identify some of the crucial thermal properties. Specifically, thermal conductivity determines the working temperature levels of the material, and it plays a critical role in the performance of materials in high temperature applications such as aerospace vehicles and space systems. It is an essential parameter in problems involving heat transfer and thermal structures (Saad et al., 2011).

Carbon-carbon is lightweight, retains its strength at high temperatures, has high tailorable thermal conductivity, and exhibits low wear from room temperature to high temperatures. These characteristics make the carbon-carbon composites attractive candidates as advanced thermal system materials (Ohlhorst et al., 1997). Primarily, the composites are employed in the aerospace industry thereby capitalizing on their auspicious thermal capabilities. Due to their excellent mechanical, thermal, wear, and frictional properties, the carbon-carbon composites are great candidates in today's brake industries in aviation and some automotive industries (Iqbal et al., 2011). Applications requiring thermal management or system elements needing high temperature stability, including rocket nozzles and exit cones, also benefit from the desirable carbon-carbon composite qualities.

Graphitization of the carbon-carbon composite involves heat treating it to a temperature of $2500^{\circ} \mathrm{C}$. This processing technique extends the composite's capabilities and alters its molecular and thermal makeup. Increasing the graphite order of the standard carbon structure, results in a significantly higher thermal conductivity than the non-graphitized composite.

The thermal properties through the thickness of the carbon-carbon composites and graphitized carbon-carbon composites were examined. These materials were developed at Center for Composite Materials and analyzed in the Thermal Characterization Laboratory at North Carolina A\&T State University as part of the NASA-URC "Center for Aviation Safety" sponsored research. The carbon-carbon composites tested were produced by the Resin Transfer Molding (RTM) process using T300 2-D carbon fabric and Primaset PT-30 cyanate ester.

Carbon-Carbon composites are used in a wide variety of fields and it is necessary to develop and retain a database of detailed thermal information about the material to ensure safe operating temperatures in factories and proper function in systems. Currently, there is limited information on the thermal characterization of carbon-carbon. Iqbal et al. (2011) investigated the effect of heat treatment on thermal properties of carbon-carbon composites and Ohlhorst et al. (1997) generated a thermal conductivity database of selected carbon-carbon and graphitized carbon-carbon materials.

\section{THERMAL DIFFUSIVITY}

Thermal diffusivity measures how quickly heat can travel through a material. It determines the working temperature levels of the material and plays a critical role in the performance of materials in high temperature applications. The thermal diffusivity of a material can be measured in several different ways. There are steady-state methods as well as transient techniques. Available procedures include Thermal Wave Interferometry (TWI), Thermographic methods, the flash method, the Hot-wire method, and others. Recently, transient techniques have been preferred in measuring thermal properties of materials, the most common of these being the flash method (Nunes dos Santos, 2007).

\subsection{The Flash Method}

W. J. Parker founded the flash method in 1961, and it is the most frequently used transient photothermal technique and has the versatility of using a lamp or laser as the energy source. In many countries it is considered a standard for thermal diffusivity measurement of solid materials (Cernuschi et al., 2004). As adopted by the United States, the laser flash method is a standard test method and is defined by the

\footnotetext{
*Corresponding Author Email: saad@ncat.edu
} 
American Society for Testing and Materials standard E-1461. It involves a small cylindrical, thin disk specimen being heated in a closed environment to a desired temperature, usually between 20 and $500^{\circ} \mathrm{C}$. Once the disk and the environment have reached the specified temperature the front face is subjected to quick radiant energy pulse as shown in Fig. 1. The energy source can be a laser or a lamp. A detector measures the resulting temperature change with respect to time on the rear face of the sample. The data acquisition system then records the temperature change of the rear face of the specimen versus time. A graphical representation of this data is called the thermogram of the flash. Figure 2 displays the theoretical model thermogram. The time in which it takes the rear face of the specimen to reach half the maximum temperature rise is called the halftime, $\mathrm{t}_{1 / 2}$.

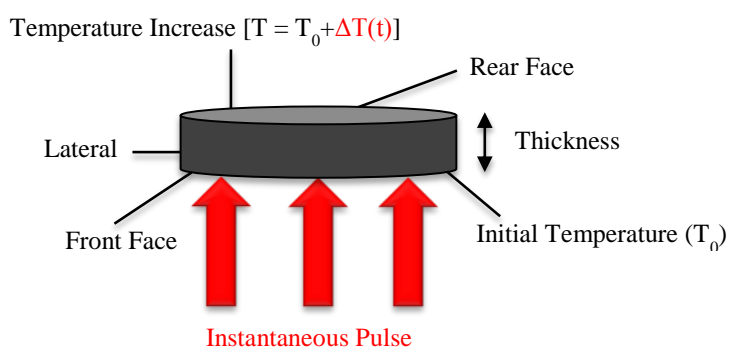

Fig. 1 Schematic of the Flash Method

Utilizing the equation for the temperature distribution within a thermally insulated solid of uniform thickness L developed by Carslaw and Jeager (1959), a mathematical expression to calculate thermal diffusivity was derived (Parker et al., 1979). An abbreviated version of this derivation is given as:

$$
\begin{aligned}
& T(x, t) \\
& =\frac{1}{L} \int_{0}^{L} T(x, 0) d x \\
& +\frac{2}{L} \sum_{n=1}^{\infty} \exp \left(\frac{-n^{2} \pi^{2} \alpha t}{L^{2}}\right) \cos \frac{n \pi x}{L} \int_{0}^{L} T(x, 0) \cos \frac{n \pi x}{L} d x
\end{aligned}
$$

where $\alpha$ is the thermal diffusivity in $\mathrm{cm}^{2} / \mathrm{s}$. If a pulse of radiant energy, $Q\left(\mathrm{~J} / \mathrm{cm}^{2}\right)$, is instantaneously and uniformly absorbed into a small depth referred to as $g$, at the front face $(\mathrm{x}=0)$ of the thermally insulated solid material (Clark and Taylor, 1975), the temperature distribution at the initial condition is given by:

$$
\begin{gathered}
T(x, 0)=\frac{Q}{\rho C_{p} g} \quad \text { for } \quad 0<x<g \\
T(x, 0)=0 \text { for } \quad g<x<L
\end{gathered}
$$

With the above initial conditions, equation 1 can be expressed as:

$$
\begin{aligned}
& T(L, t) \\
& =\frac{1}{L}\left[\int_{0}^{g} \frac{Q}{\rho C_{p} g} d x+\int_{g}^{L} 0 \cdot d x\right] \\
& +\frac{2}{L} \sum_{n=1}^{\infty} \exp \left(\frac{-n^{2} \pi^{2}}{L^{2}} \alpha t\right) \cos \frac{n \pi x}{L}\left[\int_{0}^{g} \frac{Q}{\rho C_{p} g} \cos \frac{n \pi x}{L} d x\right. \\
& \left.+\int_{g}^{L} 0 \cdot \cos \frac{n \pi x}{L} d x\right]
\end{aligned}
$$

$$
\begin{aligned}
& T(x, 0) \\
& =\frac{Q}{\rho C_{p} L}\left[1+2 \sum_{n=1}^{\infty} \exp \left(\frac{-n^{2} \pi^{2} \alpha t}{L^{2}}\right) \cos \frac{n \pi x}{L} \frac{\sin \frac{n \pi g}{L}}{\frac{n \pi g}{L}}\right]
\end{aligned}
$$

Since $\mathrm{g}$ is a very small number then it follows that $\sin (\mathrm{n} \pi \mathrm{g} / \mathrm{L}) \approx \mathrm{n} \pi \mathrm{g} / \mathrm{L}$ and $\cos (\mathrm{n} \pi \mathrm{x} / \mathrm{L})=(-1)^{\mathrm{n}}$. Once these are applied, the temperature distribution at the rear face ( $\mathrm{x}=\mathrm{L})$ is expressed as (Parker et al., 1979):

$$
T(L, t)=\frac{Q}{\rho C_{p} L}\left[1+2 \sum_{n=1}^{\infty}(-1)^{n} \exp \left(\frac{-n^{2} \pi^{2}}{L^{2}} \alpha t\right)\right]
$$

Setting

$$
T_{m}=\frac{Q}{\rho C_{p} L}
$$

where $\mathrm{T}_{\mathrm{m}}$ is the maximum temperature at the rear face. Parker et al. (1979) then defined two dimensionless parameters, $V$ and $\omega$ as:

$$
\begin{gathered}
V(L, t)=\frac{T(L, t)}{T_{m}} \\
\omega=\frac{\pi^{2} \alpha t}{L^{2}}
\end{gathered}
$$

Combining equations 6, 8, and 9 yields (Parker et al., 1979):

$$
V=1+2 \sum_{n=1}^{\infty}(-1)^{n} \exp \left(-n^{2} \cdot \omega\right)
$$

Setting $\mathrm{V}=0.5$ allows for the determination of $\omega$ at the time required for the rear face to reach half of the maximum temperature rise. Substituting $\omega=1.38$ into equation 9 allows for a mathematical equation for thermal diffusivity to be stated as (Parker et al., 1979):

$$
\alpha=0.1388 \frac{L^{2}}{t_{1 / 2}}
$$

W. J. Parker's derivation is a theoretical model of the flash method and is the ideal case. It assumes that the specimen is mostly homogeneous and isotropic, that there is one dimensional heat flow, and that there are no heat losses from the specimen (ASTM 2007). It also assumes that energy pulse is uniformly subjected across the front face of the specimen and that the pulse is instantaneous. Because of this, since Parker's original derivation, many researchers have developed correction factors. These include but are not limited to Cowan, Clark and Taylor, Koski, and Heckman (Beck and Dinwiddie, 1995). Each of these correction factors use different or a combination of methods to reanalyze the theoretical model and impose additional parameters. The Clark and Taylor (1975) correction factor accounts for radiation heat losses and is used in the research conducted in this experiment. In addition Clark and Taylor examined the thermogram at different points before the maximum temperature rise was reached and developed a correction factor. The correction factor is computed using the following equation:

$$
\begin{array}{r}
K_{R}=-0.3461467+0.361578\left(\frac{t_{0.75}}{t_{0.25}}\right)^{2} \\
-0.06520543\left(\frac{t_{0.75}}{t_{0.25}}\right)^{2}
\end{array}
$$

Specifically, they analyzed the time to reach 25 percent and 75 percent

After integration the above equation can be written as: 
of the maximum temperature change. The corrected thermal diffusivity equation as defined by Clark and Taylor is

$$
\alpha_{\text {corrected }}=\frac{\alpha K_{R}}{0.13885}
$$

\subsection{Experimental Apparatus}

In general, the ASTM standard E-1461 delineates the minimum requirements for the apparatus. The key components are the flash source, specimen holder, temperature response detector, recording device, and an environmental enclosure when testing above and below room temperature (ASTM 2007). The flash source can be any device able to emit a quick energy pulse, usually a lamp or laser. The apparatus used in this facility was purchased from the Anter Corporation and is commercialized. It is the Flashline TM 2000 and utilizes a high intensity xenon lamp as the pulse source (Anter Corporation). The pulse duration time should be less than $2 \%$ of the halftime of the specimen to be measured in order to keep the error due to finite pulse less than $0.5 \%$. The apparatus is automated and capable of testing up to four specimens in each run. The thermal property analyzer also contains a vacuumcapable environmental enclosure, in which nitrogen gas is used to evacuate the chamber. The detector should be any sensor that can measure a linear electrical output proportional to a small temperature rise. It along with its amplifier must have a response time of no more than $2 \%$ of the half-time. The temperature response InSb infrared detector outputs a linear electrical signal proportional to a small temperature change experienced by the rear face of the specimen after the pulse. The data acquisition system can be pre-programmed within one time period for the acceptable resolution of at least $1 \%$ for the quickest thermogram the system can deliver (ASTM 2007). Figure 2 shows the half time, which is the time required for the rear face of the sample to reach half of the maximum temperature rise.

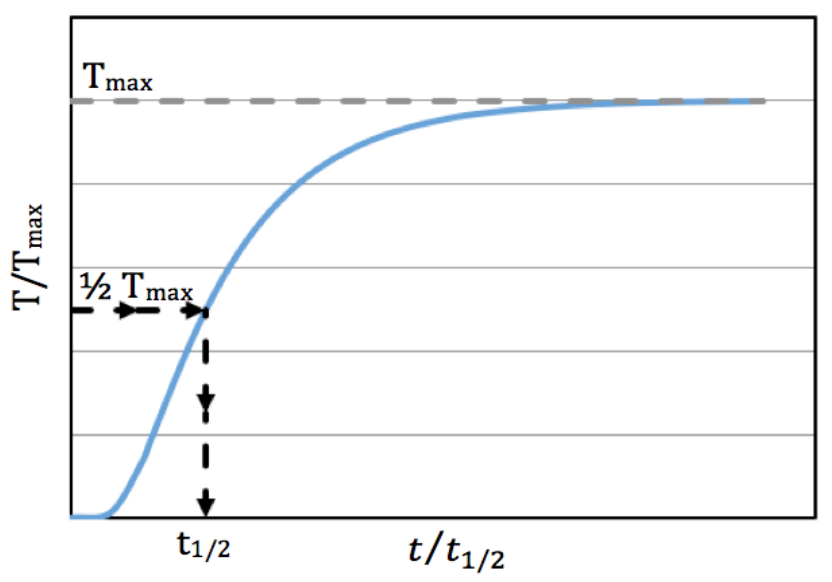

Fig. 2. Flash Method Thermogram

\subsection{Test Specimen Preparation}

The test specimen were prepared to be thin circular disks of 10 to 30 $\mathrm{mm}$ in diameter, whose front face surfaces are less than that of the energy pulse beam (ASTM 2007). According to ASTM E-1461 each specimen should be thick enough to be representative of the test material but remain close to the 1 to $6 \mathrm{~mm}$ range. Overall, the optimum thickness depends upon the magnitude of the estimated thermal diffusivity and should be chosen so that the time to reach half of the maximum temperature falls within the 10 to $1000 \mathrm{~ms}$ range. In order to accomplish these specified dimensions, a drill press equipped with a diamond plated drill bit was used to cut the material to the appropriate diameter. When necessary, the specimens were milled to achieve the preferred thickness.

Both the rear and front faces were flat and parallel within $0.5 \%$ of their thickness to maintain pulse uniformity. The standard suggests that a thin, uniform layer of graphite be applied to both faces of the specimens to improve the capability of absorbing the applied energy flash by reducing the reflection from the specimen. This was not necessary for the experiments performed in this work due to the material nature of the carbon-carbon.

\subsection{Experimental Procedure}

The experiments were conducted following the test standard ASTM E1461. $12.7 \mathrm{~mm}(0.5 \mathrm{inch})$ and $25.4 \mathrm{~mm}$ (1 inch) diameter samples were used. The diameter, thickness, mass, and density were documented. Each sample was placed in the specimen holder housed inside a vacuum seal environmental enclosure. The environmental enclosure was purged using nitrogen gas to form an inert environment for the samples. Approximately $1 \mathrm{~L}$ of liquid nitrogen was manually poured in the receptacle of the IR detector. The thickness, diameter, and mass were input into the FlashLine ${ }^{\mathrm{TM}} 2000$ System, and the test was initiated at ambient temperature. Each sample was tested to a maximum temperature of $315^{\circ} \mathrm{C}$. At each designated temperature, a minimum of three flashes were performed at a time. The results were compiled, analyzed, and necessary correction factors were applied. The time required for each experimental run varies depending on the range of temperatures tested and the temperature increment.

\section{SPECIFIC HEAT}

Specific heat signifies how much heat per unit mass is required to raise the temperature of a material one degree Celsius. Differential Scanning Calorimetry (DSC) is a common technique used to measure the specific heat of materials. This technique is based upon the measurement of the change of the difference in the heat flow of the unknown material to that of a reference sample, while they are being subjected to a controlled temperature sequence (Höhne et al., 2003). Utilizing the measured heat flow rate of the unknown sample, Differential Scanning Calorimetry can determine how a material's heat capacity varies with respect to temperature.

\subsection{Differential Scanning Calorimetry (DSC)}

Differential scanning calorimetry (DSC) is a thermo-analytical technique that is widely used for the measurement of specific heat. As accepted by the United States, its methodology is defined by ASTM standard E-1269. To conduct a differential scanning calorimetry measurement, the test specimen and reference sample are placed on a metallic block with high thermal conductivity and are enclosed in a furnace within the calorimeter. The metallic block ensures a good heatflow path between the specimen and reference. The sample and the reference are subjected to an identical temperature program. The heat capacity changes in the specimen, which leads to a difference of temperature and heat flux relative to the reference. The calorimeter measures the temperature difference and calculates heat flow from calibration data. As a result, the specific heat of the sample can be calculated using the heat flow results.

To calculate the specific heat of unknown material, the heat flux of the unknown and a reference must be measured using the differential scanning calorimeter. Using the measured heat flux values and the known specific heat of the reference, the specific heat of the unknown material can be calculated using the ratio method technique. Since the differential scanning calorimeter is at constant pressure, the change in enthalpy of the reference is equal to the heat absorbed or released in by the reference (ASTM 2005). This is depicted mathematically as: 


$$
Q=m * d h
$$

Dividing both sides of the above equation by time leads to the following relationship:

$$
\dot{Q}=m \frac{d h}{d t}=m \frac{d q}{d t}
$$

where $d q / d t$ is the heat rate and $d h / d t$ is the change of enthalpy with respect to time. At constant pressure, the relationship for specific heat can be written as:

$$
C_{p}=\left(\frac{1}{m}\right) \frac{d H}{d T}
$$

where

$$
d H=m \cdot d h
$$

Using the chain rule the equation can be rewritten as:

$$
C_{p}=\left(\frac{1}{m}\right) \frac{d t}{d T} \frac{d H}{d t}
$$

From equations 12 and 14, the specific heat can be written as:

$$
C_{p}=E\left(\frac{1}{m}\right)\left[\left(\frac{d t}{d T}\right)\left(\frac{d Q}{d t}\right)\right]
$$

where $\mathrm{E}$ is the calibration constant and $d t / d T$ is the inverse temperature distribution over time. Using the ratio method, equation 15 can be written for the reference material as:

$$
C_{p_{r e f}}=E\left[\left(\frac{1}{m_{r e f}}\right)\left(\frac{d t}{d T}\right)\left(\frac{d Q}{d t}\right)_{r e f}\right]
$$

Rearranging the calibration constant can be expressed by:

$$
E=\frac{C_{p_{r e f}} m_{r e f}}{\left(\frac{d t}{d T}\right)\left(\frac{d Q}{d t}\right)_{r e f}}
$$

The specific heat for the unknown material can be given by substituting equation 17 into equation 15

$$
C_{p}=\left(\frac{C_{p_{r e f} m_{r e f}}}{\left(\frac{d t}{d T}\right)\left(\frac{d Q}{d t}\right)_{r e f}}\right)\left[\left(\frac{1}{m}\right)\left(\frac{d t}{d T}\right)\left(\frac{d Q}{d t}\right)\right]
$$

Reducing like terms in equation 19 , the specific heat of the unknown material can be written as:

$$
C_{p}=\left(C_{p_{r e f}}\right)\left(\frac{m_{r e f}}{m}\right)\left[\frac{(d Q / d t)}{(d Q / d t)_{r e f}}\right]
$$

\subsection{Experimental Apparatus}

The calorimeter used in this research is the DSC 200 F3 Maia®, Differential Scanning Calorimeter manufactured by NETZSCH. It is a heat flux system that combines high stability, high resolution, and fast response time throughout a substantial temperature range. With the addition of the Intracooler 40, the temperature range extends from ambient temperature to cryostatic temperatures covering a larger temperature spectrum $\left(-40^{\circ} \mathrm{C}\right.$ to $\left.600^{\circ} \mathrm{C}\right)$. The heating rate is adjustable from as low as $0.001 \mathrm{~K} / \mathrm{min}$ to as high as $100 \mathrm{~K} / \mathrm{min}$ while keeping a temperature accuracy of $0.1 \mathrm{~K}$ (NETZSCH 2008).

The DSC 200 F3 Maia ${ }^{\circledR}$ Differential Scanning Calorimeter consists of a furnace block, sample chamber, cooling system, heat flux sensor, and a purge gas capability. The furnace block contains a miniature jacketed heater that provides the source of heat during the experiment. The furnace temperature is measured by a thermocouple integrated into the furnace walls. The sample chamber is sealed within the instrument's lid, and has two additional lids to prevent a contamination from outside sources. The system's temperature is reduced using compressed air. This is provided by an additional device, the Intracooler 40. The calorimeter uses a high sensitivity type E heat flux sensor for its measurements (NETZSCH 2008).

\subsection{Test Specimen Preparation}

A good thermal contact between the heat flux sensor and the sample is essential for optimum results. To achieve this, the sample should be oriented such that it lays as flush as possible with the bottom of the aluminum crucible. A 4-mm or 6-mm diameter and 1-mm thick sample can be used with this equipment using the corresponding crucible size. Every sample was weighed three times, and the average mass was documented. Each sample was placed into the crucible, and a lid was positioned on top of the crucible to fully enclose the sample. Using tweezers, the crucible was then carefully placed on the heat flux sensor making sure the crucible was centered on the sensor.

\subsection{Experimental Procedure}

The differential scanning calorimetry experiment was performed following the guidelines in testing standard ASTM E-1269, standard test method for determining specific heat. The differential scanning calorimeter and data acquisition system were initialized and were allowed to reach thermal equilibrium. During this period the apparatus was purged with argon gas at a rate of $50 \mathrm{~mL} / \mathrm{min}$ to produce an inert testing atmosphere. To measure the specific heat of a sample, a minimum of three runs must be performed.

Before the specific heat of the carbon-carbon composites was determined, a temperature calibration and baseline for each material were performed. Since the samples were placed inside an aluminum crucible for testing, the crucible added a contact resistance to samples. The baseline helps to correct this contact resistance, thereby increasing the accuracy of the results. The initial baseline run was performed by placing two empty crucibles in the designated location on heat flux sensor, as seen in Fig. 3. The furnace was heated to the designated initial temperature of the program, and held there isothermally for at least four minutes while the calorimeter recorded the thermal curve. The crucibles were heated to the final temperature at rate of $20^{\circ} \mathrm{C} / \mathrm{min}$ and held isothermally again, while the calorimeter recorded the thermal curve.

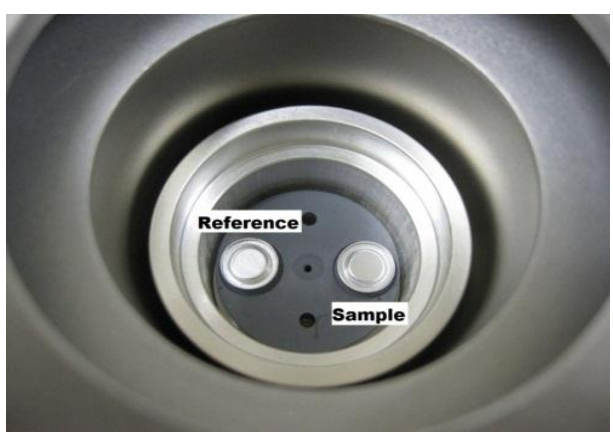

Fig. 3. Crucible Placed on the Heat Flux Sensor 
Following the baseline run, the calorimeter testing chamber was cooled to ambient temperature. The crucible on the reference location in the testing chamber was replaced with a sapphire reference. After applying the previous baseline to correct for the aluminum crucible, the same temperature program used for the baseline was executed for the sapphire reference. The measured specific heat of the sapphire was compared to the known specific heat value for sapphire to determine the error. The test was repeated for the carbon composite samples. To verify that the baseline did not alter, a baseline was established after every fourth test. Using the measured sapphire as a reference, the ratio method was used to determine the specific heat of the carbon-carbon composites.

\section{Results and Discussions}

The flash method was used to determine the thermal diffusivity through the thickness of the carbon-carbon composites. Two types were investigated, non-graphitized carbon-carbon and graphitized carboncarbon. Figure 4 displays a micrograph image of the material used in this investigation.

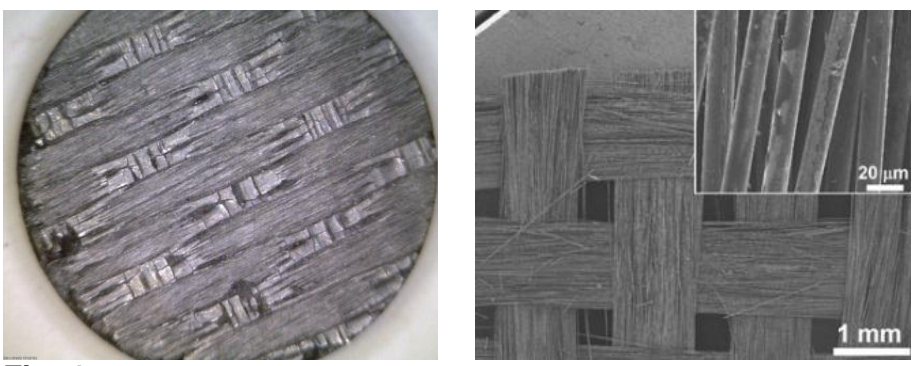

Fig. 4. Micrograph Image of the Carbon - Carbon Composite

The thermal diffusivity of the carbon-carbon composites was measured between room temperature and $315^{\circ} \mathrm{C}$. This range was selected due to the temperature limitations of the apparatus.

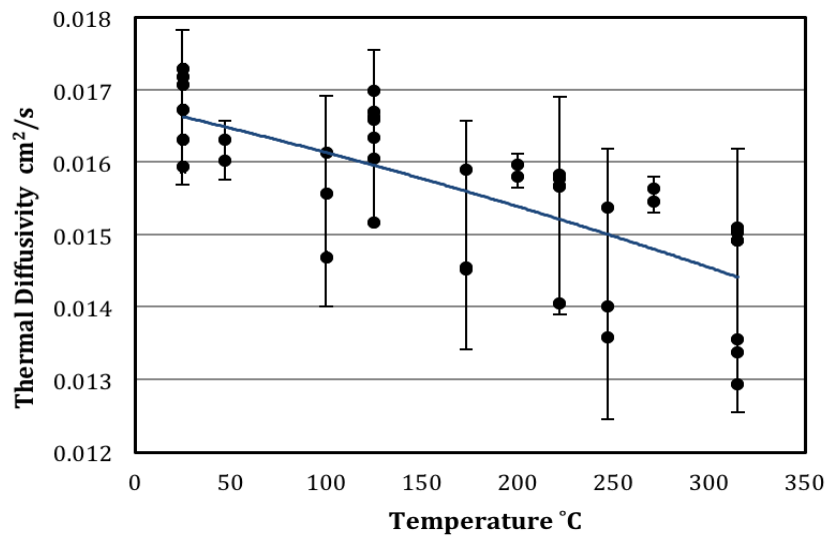

Fig. 5. Thermal Diffusivity of Carbon - Carbon Composite

Figure 5 displays a magnified view of the error bars of the thermal diffusivity values of the carbon-carbon composite. In general, temperature has a minimum effect on the thermal diffusivity of carboncarbon where the values drop approximately $10 \%$ over the temperature range. As shown in Fig. 6 the thermal diffusivity of the graphitized carbon-carbon dropped nearly $50 \%$ from room temperature to $315^{\circ} \mathrm{C}$. The thermal diffusivity of the graphitized carbon-carbon was more influenced by the temperature because the difference in the coefficient of thermal expansion between the matrix resin and fiber thermal behavior at higher temperatures becomes more apparent resulting in a sharp decrease in thermal diffusivity (Iqbal et al., 2011). The effect of the heat treatment on the graphitized material has already caused a permanent change on the matrix and the fiber. This effect is increased when the material undergoes testing at temperatures above room temperature. In contrast the non-graphitized material did not experience a heat treatment, therefore testing at higher temperatures does not create such a dramatic change on the thermal diffusivity as shown in Fig. 7.

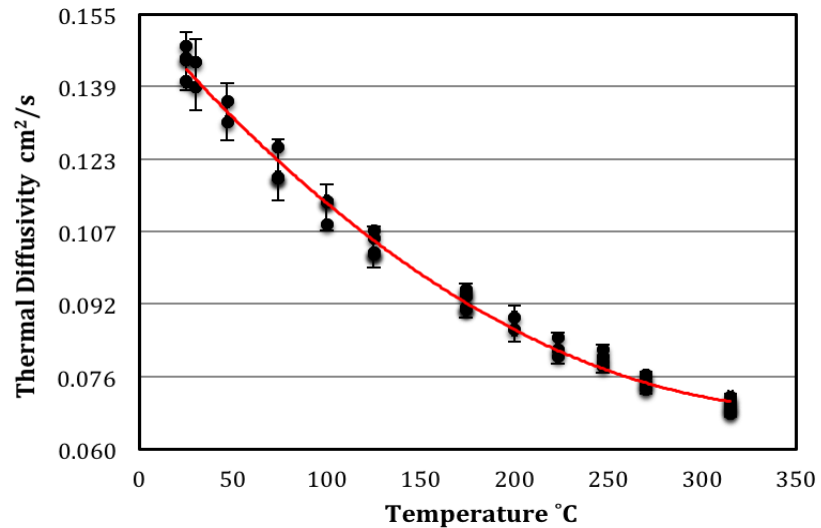

Fig. 6. Thermal Diffusivity of Graphitized Carbon-Carbon Composite

A comparison of the graphitized and non-graphitized trend lines in Fig. 7 further shows that the diffusivity values of the graphitized carbon-carbon are 8.75 times those of the non-graphitized composites. The large difference between the thermal diffusivity values is due to the effect of graphitization on the composite.

Graphitization is the transformation of a standard carbon structure into a higher ordered graphite structure. The order increase can be observed as a shift from an amorphous carbon structure to a sequence of stacked parallel plates. The graphitization process takes place at temperatures greater than $2500^{\circ} \mathrm{C}$. The structural shift begins slowly near $1800^{\circ} \mathrm{C}$. Hydrogen, Sulfur, and other impurities abscond from the material between $1200^{\circ} \mathrm{C}$ and $2000^{\circ} \mathrm{C}$. Eventually, the carbon crystals grow from $5 \mathrm{~nm}$ to $100 \mathrm{~nm}$ or larger. Additionally, the spacing between the carbon layers begins to decrease and density increases. It is known that a decreased structural order will tend to significantly reduce the thermal conductivity of a material. Because the structural order of the graphitized samples is increased this causes them to have a significantly higher thermal diffusivity than the non-graphitized composites as apparent in Fig. 7. The maximum thermal diffusivity occurs at room temperature. The measured experimental values of thermal diffusivity in this research are similar to those found for comparable materials in Ohlhorst et al. (1997).

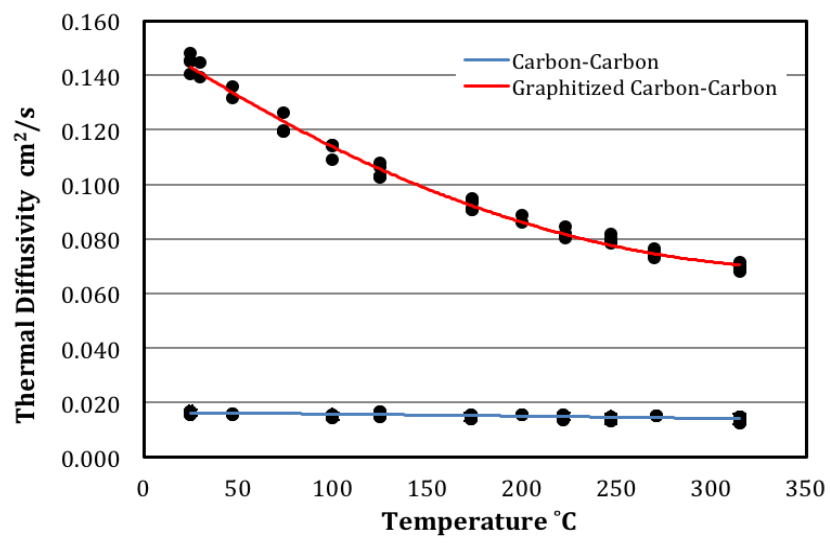

Fig. 7. Thermal Diffusivity of Tested Materials 
One will also observe that the error bars of the carbon-carbon composites are much smaller than the error bars of the graphitized carbon-carbon composites. This occurs because the graphitized carboncarbon is much more conductive than the non-graphitized carboncarbon. The increased diffusivity of the graphitized composites allows the energy pulse to propagate through the material much faster resulting is less radiation heat loss from the sample and therefore less error.

The error was calculated at each temperature, and the error bars shown in Figs. 5 and 6 indicate a two standard deviation range about the associated data. This range depicts the interval of values in which $95 \%$ of the thermal diffusivity measurements should lie. It can be observed that there is much less error associated with the graphitized carbon-carbon diffusivity values.

Population data sets provide the statistical estimates known as the population mean value and the population standard deviation defined by (Figliola and Beasley, 2011)

$$
\sigma=\sqrt{\frac{1}{N} \sum_{i=1}^{N}\left(a_{i}-\bar{a}\right)^{2}}
$$

The results from each temperature tested are considered as a population, where $N$ is the total number of measurements, $a_{i}$ represents a single $\mathrm{i}^{\text {th }}$ measurement, and $\bar{a}$ is the mean value of the data at each temperature. Additionally, the margins of errors were calculated for each temperature using standard error of the mean, as shown in Eq. (25).

$$
S E=\frac{\sigma}{\sqrt{\bar{N}}}
$$

The critical value from normal distribution chart based on $95 \%$ confidence is 1.96 , and the margin of error, $e$, is determined by the following

$$
e= \pm 1.96 \cdot S E
$$

The percent error can now be found using Eq. (27).

$$
\operatorname{error} \%=\frac{e}{\text { mean }}
$$

According to the ASTM E1461 (2005) testing standard, the optimum thickness of test specimen should be selected such that the time to reach half of the maximum temperature (half-time), $t_{1 / 2}$ falls within the 10 to $1000 \mathrm{~ms}(0.01$ to $1 \mathrm{~s})$ range. To verify that the samples were fabricated to the proper thickness, an initial test was preformed to analyze the half-times of the test samples. The half-times reached at each temperature during this experiment were recorded and can be found in Fig. 8. The documented half-times for each material at the specified temperature were within the acceptable range as defined by the testing standard and shown in Fig. 8. This signifies that an appropriate thickness was chosen for the test samples. It can be observed that the half-times of the graphitized carbon-carbon composite are nearly an order of magnitude smaller than those of the carboncarbon composite. The graphitized carbon-carbon composites have a shorter half-time because they are more conductive which allows the energy pulse to propagate through the material much faster than in the non-graphitized carbon-carbon.

The temperature and time data can also be analyzed using thermogram curves. The normalized thermogram curves can be compared to the theoretical model in order to depict and explain shape differences. Figure 9 shows the thermogram temperature curves for the carbon-carbon composite and the theoretical model. As the ratio of time to halftime increases it can be observed that the experimental temperature curves differ more and more from the theoretical model.
These deviations are due to radiation heat losses. It can be observed that at lower temperatures (i.e. cryogenic), there are significant losses in comparison to those losses experienced as higher temperatures.

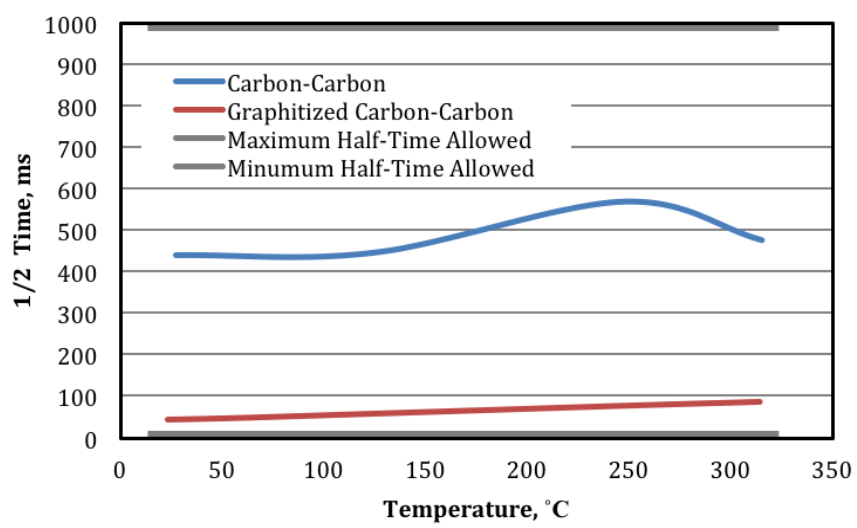

Fig. 8. Half-Time of Test Materials Comparison to Allowable Limits

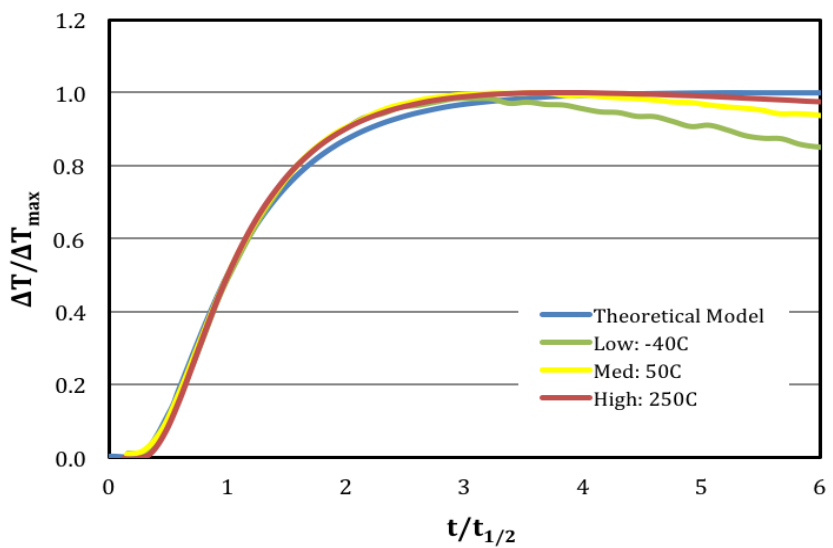

Fig. 9. Comparison of the Carbon-Carbon Thermogram Curves to the Theoretical Model

Overall there is an inverse correlation, the higher the test temperature the lower the amount of radiation heat loss $\left(\mathrm{T}^{4}(\mathrm{t})-\mathrm{T}_{\infty}^{4}\right)$ where $T(t)$ corresponds to the temperature of the sample after the instantaneous energy pulse. Figure 9 shows the thermogram for $\mathrm{T}_{\infty}=-$ $40^{\circ} \mathrm{C}, 50^{\circ} \mathrm{C}, 250^{\circ} \mathrm{C}$. $\mathrm{T}_{\infty}$ represents the initial temperature of the sample as well as the ambient temperature immediately before the flash occurs.

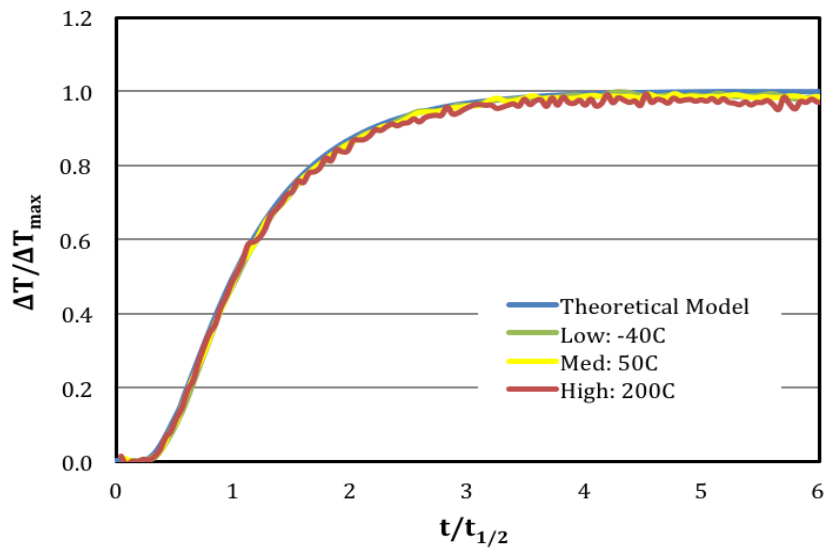

Fig. 10. Comparison of the Graphitized Carbon-Carbon Thermogram Curves to the Theoretical Model 
Therefore at a higher temperature testing environment the radiation heat loss is less than the radiation heat loss at a lower test environment temperature. In regards to the graphitized carbon-carbon composite curves in Fig. 10, the radiation heat loss is minimal for all of the temperatures tested. The graphitized test specimen experienced less radiation heat loss because the energy pulse travels very quickly through the materials allowing less radiation losses from the material.

The specific heat measurements of the carbon-carbon and graphitized carbon-carbon composites show a trend that is extremely close to linear. A comparison of the results can be found in Fig. 11.

Utilizing the density, specific heat, and thermal diffusivity data, the thermal conductivity through the thickness of the composites was calculated using the following equation:

$$
k=\rho c_{p} \alpha
$$

The resulting thermal conductivity values of the carbon-carbon composites are compared in Fig. 12. The thermal conductivity of the graphitized carbon-carbon composite is an order of magnitude higher than that of the non-graphitized. The increase in thermal conductivity is due to the increase in the crystallinity of the graphitized material when compared to the non-graphitized material (Iqbal et al., 2011). The thermal conductivity results obtained in this investigation are similar to those found for comparable materials in Ohlhorst et al. (1997).

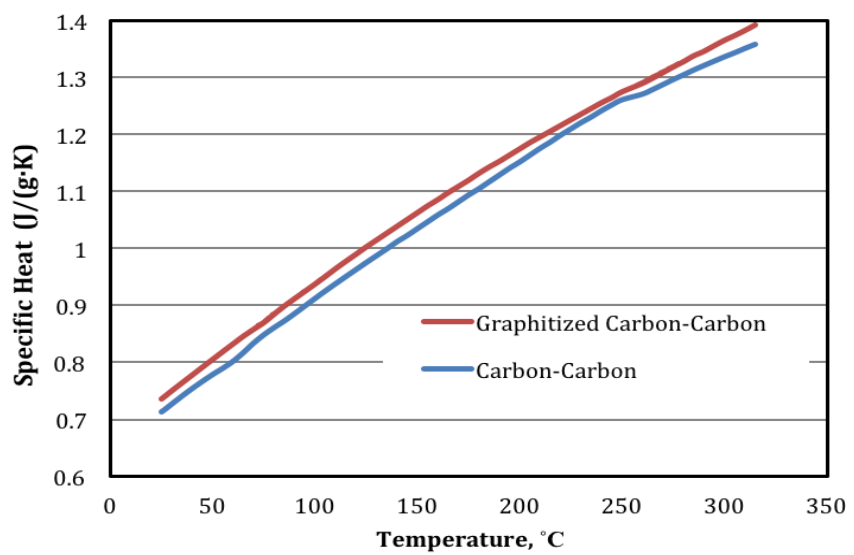

Fig. 11. Specific Heat of Tested Materials

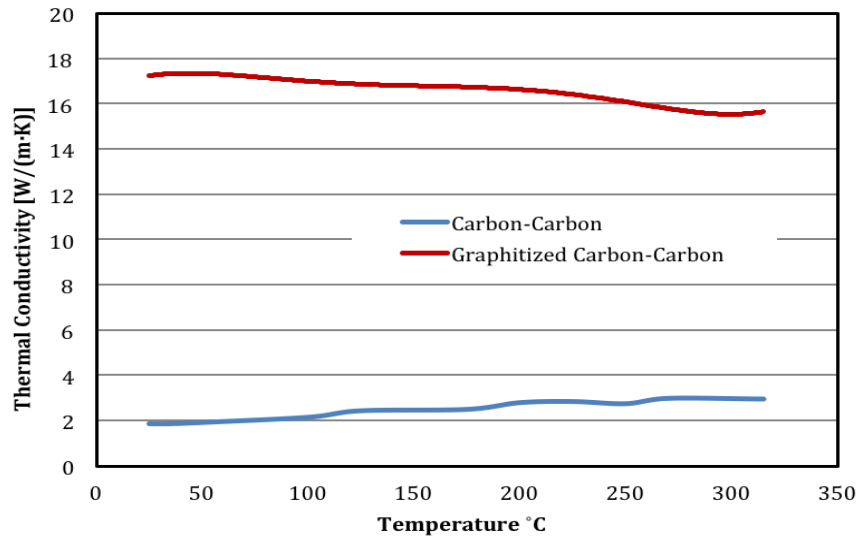

Fig. 12. Thermal Conductivity Comparison of Carbon-Carbon and Graphitized Carbon-Carbon Composites

Table 1 gives a summary of the thermal property results at room temperature $\left(25^{\circ} \mathrm{C}\right)$.
Table 1. Thermal Properties at Room Temperature

\begin{tabular}{|l|c|c|}
\hline \multicolumn{1}{|c|}{ Thermal Properties } & $\begin{array}{c}\text { Carbon- } \\
\text { Carbon } \\
\text { Composite }\end{array}$ & $\begin{array}{c}\text { Graphitized } \\
\text { Carbon-Carbon } \\
\text { Composite }\end{array}$ \\
\hline Density $\left(\mathrm{g} / \mathrm{cm}^{3}\right)$ & 1.59 & 1.62 \\
\hline Specific Heat $(\mathrm{J} / \mathrm{g} \cdot \mathrm{K})$ & 0.7130 & 0.7354 \\
\hline Thermal Diffusivity $\left(\mathrm{cm}^{2} / \mathrm{s}\right)$ & 0.0162 & 0.1454 \\
\hline Thermal Conductivity $(\mathrm{W} / \mathrm{m} \cdot \mathrm{K})$ & 1.84 & 17.2 \\
\hline
\end{tabular}

\section{CONCLUSIONS}

This paper examined the thermal properties through the thickness of carbon-carbon composites from room temperature to $315^{\circ} \mathrm{C}$. The graphitized material exhibited different thermal properties than the nongraphitized material due to the effect of the heat treatment. The thermal diffusivity was measured using the flash method. Analyses were performed to validate the accuracy of the thermal diffusivity results. The DSC was used to measure the specific heat of the materials. The specific heat of the composites was determined using the heating curve of the differential scanning calorimeter. The thermal conductivity was determined using the density, specific heat, and thermal diffusivity of the composites. Due to the increased crystallinity of the graphitized material during the heat treatment the thermal conductivity of the graphitized material is greater than that of the non-graphitized.

\section{ACKNOWLEDGEMENTS}

The authors would like to acknowledge NASA-URC-Center for Aviation Safety (CAS) (Grant\# NNX09AV08A), the Center for Composite Materials Research (CCMR) at North Carolina A\&T State University, and the Oak Ridge National Laboratory's High Temperature Materials Laboratory (HTML) sponsored by the U.S. Department of Energy, Office of Energy Efficiency and Renewable Energy, Vehicle Technologies Program

\section{NOMENCLATURE}

$\bar{a} \quad$ measurement mean

$a_{i} \quad \mathrm{i}^{\text {th }}$ measurement of the sample

$C_{p} \quad$ specific heat $(\mathrm{J} / \mathrm{kg} \cdot \mathrm{K})$

E calibration constant

$h \quad$ specific enthalpy $(\mathrm{J} / \mathrm{kg})$

$k \quad$ thermal conductivity $(\mathrm{W} / \mathrm{m} \cdot \mathrm{K})$

$K_{R} \quad$ correction factor

$L \quad$ sample thickness (cm)

$m \quad$ mass $(\mathrm{kg})$

$N \quad$ number of measurements

$Q \quad$ Heat (J)

$s_{x} \quad$ sample standard deviation

$t \quad$ time (s)

$T \quad$ temperature $(\mathrm{K})$

$V \quad$ dimensionless quantity

$x \quad$ distance (m)

Greek Symbols

$\alpha \quad$ thermal diffusivity $\left(\mathrm{cm}^{2} / \mathrm{s}\right)$

$\Delta \quad$ differential quantity

$\rho \quad$ density $\left(\mathrm{kg} / \mathrm{m}^{3}\right)$

$\omega$ dimensionless quantity

Subscripts

ref reference

$0 \quad$ initial time step

$1 / 2$ half

$F \quad$ final time 


\section{REFERENCES}

ASTM Standard E1461, 2007, "Standard Test Method for Thermal Diffusivity by the Flash Method," ASTM International, West Conshohocken, PA.

ASTM Standard E1269, 2005, "Standard Test Method for Determining Specific Heat Capacity by Differential Scanning Calorimetry," ASTM International, West Conshohocken, PA.

Anter, "FlashLine ${ }^{\mathrm{TM}}$ Thermal Diffusivity Measuring System Operation and Maintenance Manual Part 1 Flashline 2000".

Beck, J. V., and Dinwiddie, R. B., 1995, "Parameter estimation method for flash thermal diffusivity with two different heat transfer coefficients," Proceedings of 23rd International Thermal Conductivity Conference, Nashville, TN.

Carslaw H. S., and Jaeger J. C., 1959, Conduction of Heat in Solids, Oxford University Press, New York.

Clark, L. M., and Taylor R. E., 1975, "Radiation Loss in the Flash Method for Thermal Diffusivity," Journal of Applied Physics, 46(2), 714-719.

http://dx.doi.org/10.1063/1.321635

Cernuschi, F., P.G. Bison, A. Figari, S. Marinetti, E. Grinzato, 2004, "Thermal Diffusivity Measurements by Photothermal and Thermographic Techniques," International Journal of Thermophysics, 25(2), 439-457.

http://dx.doi.org/10.1023/B:IJOT.0000028480.27206.cb

Cernuschi, F., P.G. Bison, A. Figari, S. Marinetti, E. Grinzato, L. Lorenzoni, 2002, "Comparison of Thermal Diffusivity Measurement Techniques," Quantitative InfraRed Thermography Journal, 28, 211221.
Figliola, R., and Beasley, D., Theory and Design for Mechanical Measurements, John Wiley \& Sons, 2011.

Höhne, G., Hemminger, W. F., and Flammersheim, H. J., 2003, Differential Scanning Calorimetry, Springer, Germany.

Nunes dos Santos, W., 2007, "Thermal Properties of Polymers by NonSteady-State Techniques," Polymer Testing, 26(4), 556-566. http://dx.doi.org/10.1016/j.polymertesting.2007.02.005

Iqbal, S. S., Dinwiddie, R., Porter, W., Lance, M., and Filip, P., 2011, "Effect of Heat Treatment on Thermal Properties of Pitch-Based Carbon Fiber and Pan-Based Carbon Fiber Carbon-Carbon Composites," Mechanical Properties and Performance of Engineering Ceramics and Composites VI, 32, 245-254. http://dx.doi.org/10.1002/9781118095355.ch23

NETZSCH, 2008, “Technical Data Sheet DSC 200 F3 Maia”.

NETZSCH, 2008, “Operating Instructions DSC 200 F3 Maia”.

Ohlrost, C. W., Vaughn, W. L., Ransone, P. O., and Tsou, H.-T., 1997, "Thermal conductivity database of various structural carbon-carbon composite materials," NASA technical memorandum, 4787, 1-96.

Parker, W. J., Jenkins, R. J., Butler, C. P., and Abbott, G. L., 1961, "Flash Method of Determining Thermal Diffusivity Heat Capacity and Thermal Conductivity," Journal of Applied Physics, 32(9), 1679-1685. http://dx.doi.org/10.1063/1.1728417

Saad, M., Baker, D., and Reaves, R., 2011, "Thermal Characterization of Carbon-Carbon Composites," IMECE2011-64061, Proceedings of 2011 ASME International Mechanical Engineering Congress and Exposition, Denver, CO.

http://dx.doi.org/10.1115/IMECE2011-64061 